\title{
Familial amyloidotic polyneuropathy in Sweden: a pedigree analysis
}

Ulf Drugge, Rune Andersson, Farideh Chizari, Mats Danielsson, Gösta Holmgren, Ola Sandgren, Alda Sousa

Abstract

Extended genealogical studies were performed on the heredity patterns in Swedish patients with familial amyloidotic polyneuropathy (FAP) using Swedish historical archives. The population studied included 239 patients: 109 patients were linked to five large pedigrees and 80 patients belonged to 30 smaller pedigrees or nuclear families. In the remaining 50 cases, no genealogical links were found. Differences in mean ages of onset between the different pedigrees were found, although a considerable variation within the pedigrees was also present. There was a tendency for later ages of onset among older generations than younger ones: descendants of affected mothers seem to be more prone to anticipation in age of onset than descendants of affected fathers. Furthermore, there seems to be a tendency for earlier ages of onset among patients with a carrier mother than a carrier father. Some extended pedigrees, from the Skellefteå and Piteå areas, are presented in detail. The former go back into the middle of the $17^{\text {th }}$ century. One important conclusion is that the mutational event may have occurred in late mediaeval times.

(f Med Genet 1993;30:388-92)

Familial amyloidotic polyneuropathy (FAP) is an autosomal dominant disease, characterised by polyneuropathy with sensory and motor disturbances, widespread autonomic dysfunction, and in some patients typical vitreous opacities. FAP type 1 was first described by Andrade ${ }^{1}$ in 1952 in Portugal. Since then more than 1000 cases of FAP belonging to more than 400 families have been reported from Portugal, and more than 300 patients belonging to at least 40 families are known in Japan. ${ }^{23}$ Since the first Swedish patients were reported in 1968 more than 230 cases have been diagnosed. The FAP populations in Portugal, Japan, and Sweden have the same mutation of transthyretin (TTR), with a substitution of methionine for valine at position 30 (TTR Met-30). ${ }^{4-6}$

The aims of the present paper are to describe and analyse the major pedigrees, based on data from Swedish historical archives, and to contribute to the discussion of a genetic model for age of onset in FAP.

\section{Patients}

Patients with the diagnosis of amyloidosis were searched for in the registers of the Departments of Medicine in Umeå and Skellefteå and the Department of Neurology, Umea over the period 1976 to 1990 . As the diagnosis of amyloidosis is dependent on histological examination of biopsy specimens, the yearly histopathological registers at the Department of Pathology were searched. In cases with amyloidosis, clinical screening of the type of amyloidosis was made based on information from clinical records. A protocol for individual clinical and family data was established for all patients.

The population studied included 239 patients, 157 males and 82 females. In 50 cases, no affected relatives or links to other FAP families were found, but 109 patients were linked to five large pedigrees, each with at least 10 patients. The remaining 80 patients belonged to 30 smaller pedigrees or nuclear families. A selection of five pedigrees will be presented in detail. RFLP (restriction fragment length polymorphism) analysis or PCR (polymerase chain reaction) were performed in 78 patients, identifying heterozygotes and homozygotes with the TTR Met-30 mutation.

\section{Genealogical methods}

The genealogical studies were primarily based on parish registration converted into microfiches and available at the Research Archives at Umeå University and unique to Sweden and Finland. Many of the registers go back to the end of the 17 th century based on a Swedish Church Law of 1686 that made the vicar of every parish responsible for registration. The principal task was to keep catechetical meeting records in order to promote the ability to read and teach basic religious tenets of the Protestant State Church. These Catechetical Meeting Records, as well as birth, marriage, and death registers, make it possible to establish family links between people living more than 200 years ago and those of the recent past.

From information collected about ancestors of probands, the ancestors of FAP families were traced back as far as possible. Owing to the relative immobility of the population long ago, close links among people are not uncommon in rural areas, and could explain the consistency of some pedigrees. The reliability of each pedigree presented in this paper can be viewed as relatively high. There are mostly few generations involved in each of the familial links, and many familial links are normally present in each pedigree. 


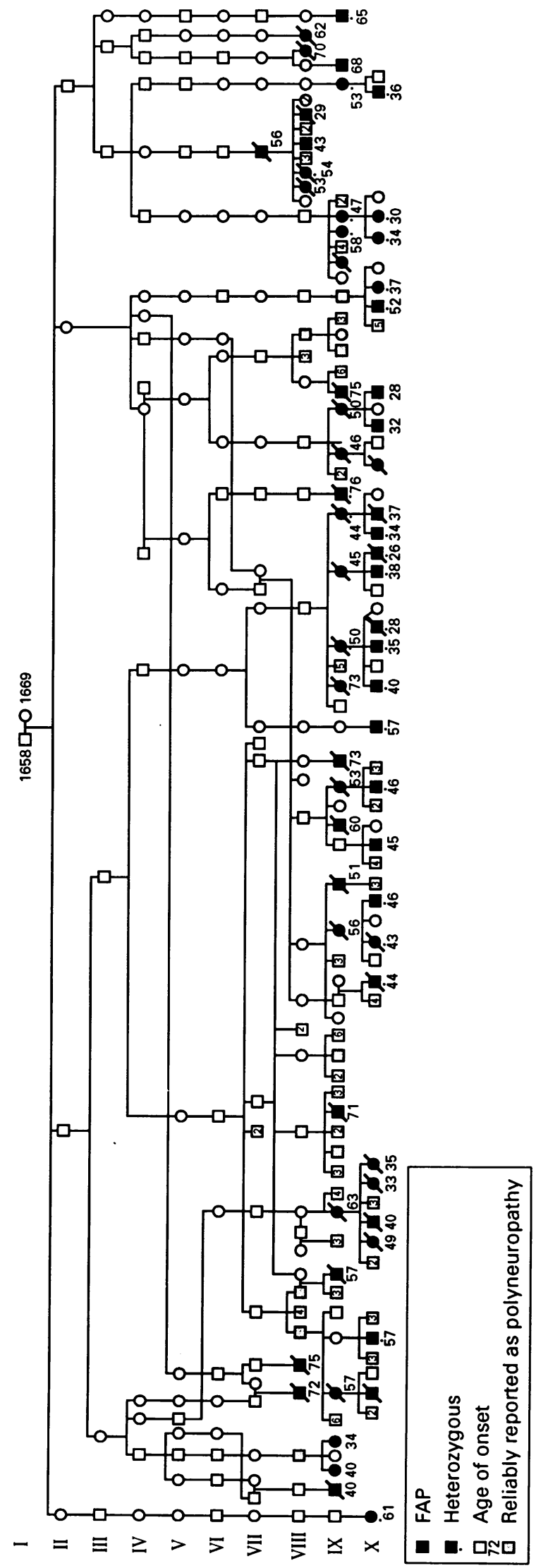

Figure 1 Pedigree $S K-1$.
Swedish FAP pedigrees have already been published by Andersson. ${ }^{7}$ Therefore, the first strategy was to build upon these results in order to search for further family extensions from his pedigrees. In one well known FAP family (Andersson, ${ }^{2}$ family 2) 20 FAP cases were already known at that time. By linking all these cases back in time to ancestors of other cases it was possible to extend this pedigree which now includes 63 patients (pedigree SK1, fig 1).

Our genealogical work on the Skelleftea (SK) area was much facilitated by a CDROM, an optic disc created at the Demographic Data Base, Umeå University. It includes data about all people registered in parish books of Skellefteå during the period 1725 to 1895 . The second strategy was a computer based search for links back in time, based on the CD-ROM (pedigrees SK-2 and SK-3, figs 2 and 3 ).

The search for ancestors of FAP patients outside the Skelleftea area was made in a manual way, using the parish registers. Further pedigrees, most of them from the Pitea (PT) area, were then constructed (pedigrees PT-2, PT-4, and PT-5, figs 4 and 5).

\section{Statistical methods}

The unpaired $t$ test was used to compare mean age of onset in descendants of a carrier father and a carrier mother, and the paired $t$ test when comparing mean differences in age of onset in parent-offspring pairs. The intraclass correlation coefficient was calculated by a random effects model of analysis of variance and the relationship between parents and their offspring's ages of onset was calculated by Pearson's correlation coefficient.

\section{Results \\ PEDIGREE ANALYSIS \\ $S K-1$}

SK-1 is the most extended pedigree of FAP families reported so far in the world. Sixtythree FAP patients in 19 nuclear families are connected to a couple living at the end of the 17 th century and the beginning of the 18th century in a village not far from what later became the city of Skellefteå.

The CD-ROM has provided possibilities for some extended tests on the validity of this pedigree. By using random samples of probands born in the Skelleftea area at the end of the 19th century, and by tracing their ancestors eight generations back in time, it was possible to note the number of common links existing among the ancestors of these probands. In fact, the number of FAP patients represented in the SK-1 pedigree (63 out of 127 registered patients in this region) is far bigger than could be expected from any random sample of linkage of common ancestors of probands in this area. To conclude, even if SK-1 includes some weak parts of multigenerational ancestral lines, the main part of the SK-1 pedigree includes probable familial links 


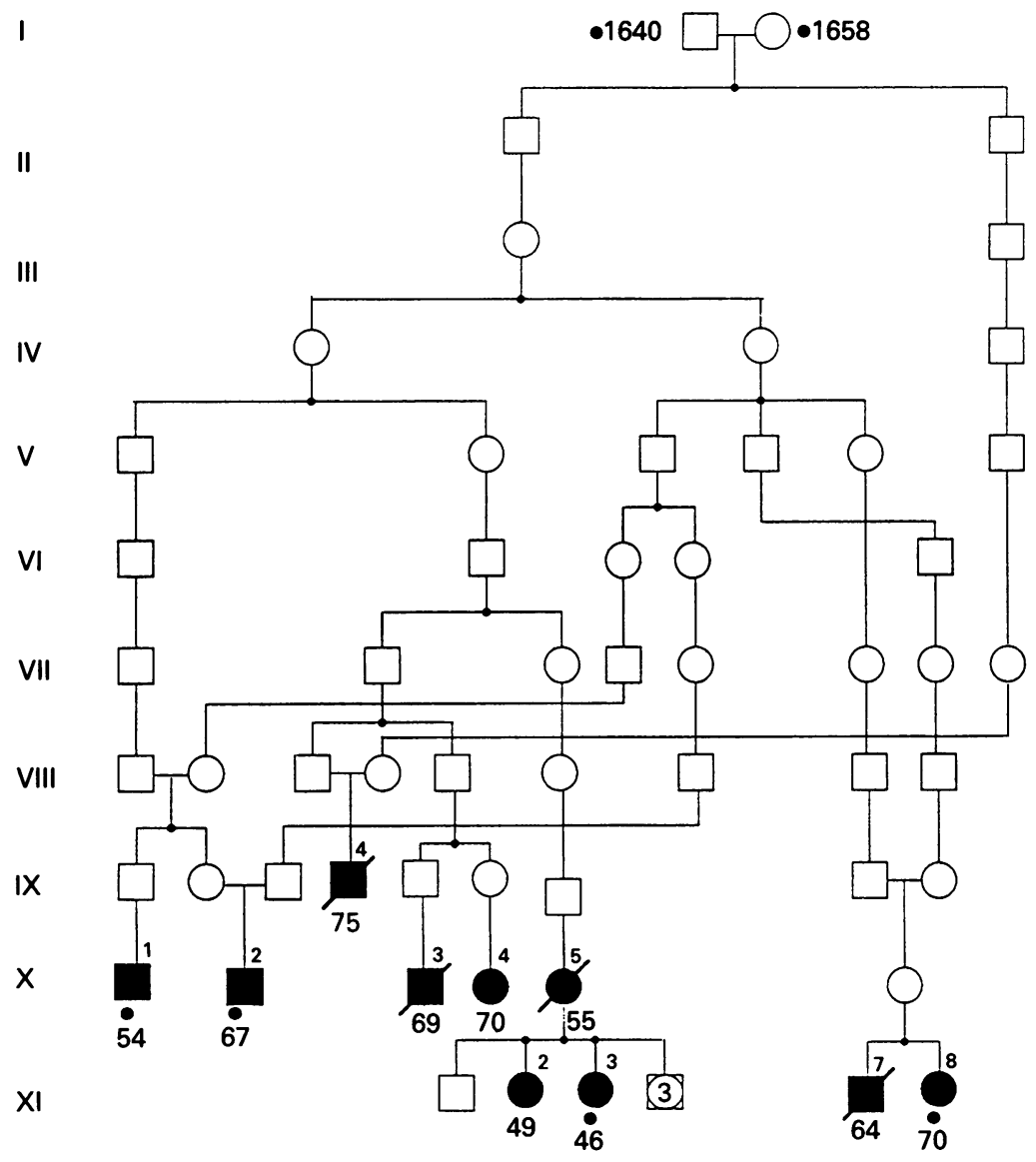

Figure 2 Pedigree $S K-2$. between FAP patients from the Skelleftea area.

Some of the ancestral lines are genetically and genealogically less important. For instance, links with no ramifications five or more generations back in time are of minor analytical importance, while others might provide us with empirical material for far reaching conclusions.

There are some general points of interest in this pedigree. The mean age of onset is some seven years lower than the Swedish mean age of onset of FAP. The variation in ages of onset is relatively high in this pedigree, ranging from 26 to 76 years (table). Various segments of the families in the pedigree have low age of onset and there are also remarkable differences between generations: older generations have a tendency for later age of onset than younger ones.

\section{$S K-2$}

Most segments of pedigree SK-2 are relatively closely related, and include a fairly complicated structure of links between 10 patients (five males, five females). They represent seven different nuclear families, among which four constitute two different cousinships. Besides some alternative links from case IX 4 to earlier generations, the links through the common ancestors to their cousins seem to be the probable ancestral ones. This reasoning indicates that the link from subject II. 2 through VII $\cdot 8$, and through VIII 4 to IX 4 should be ignored.

A general impression from this pedigree is the concise picture it gives of the distribution of age of onset. Apart from the sibs XI.2 and $\mathrm{XI} \cdot 3$ with early onset, all patients have an onset close to or somewhat later than the mean of the FAP population. However, also to be noted is the late onset age of patient $\mathrm{X} \cdot 2$ (who presented with vitreous opacities) compared to his cousin $\mathrm{X} \cdot 1$, with a difference of 13 years.

$S K-3$

This pedigree involves 10 patients representing seven nuclear families, but with common links only four generations or more back in time. The mean age of onset of the patients in the pedigree is 58 years (range 34 to 75 years), slightly higher than the general mean age of onset for the Swedish FAP population (table).

There are some striking differences in age of onset among sibs in the pedigree. The difference in age of onset between patient VII.9 and VII. 10 and between patient VIII. 6 (who presented with vitreous opacities) and VIII.7 is as much as 23 and 24 years respectively.

\section{$P T-2$ and $P T-4$}

The extended pedigree $\mathrm{PT}-2 / \mathrm{PT}-4$ can be seen as two separate pedigrees. The links between PT-2 and PT -4 are of minor importance as an analytical base. They will be treated separately here.

PT-2 consists of two affected brothers out of

Figure 3 Pedigree $S K-3$. 
II

III

IV

V

VI

VII

Figure 4 Pedigree PT-2 and PT-4

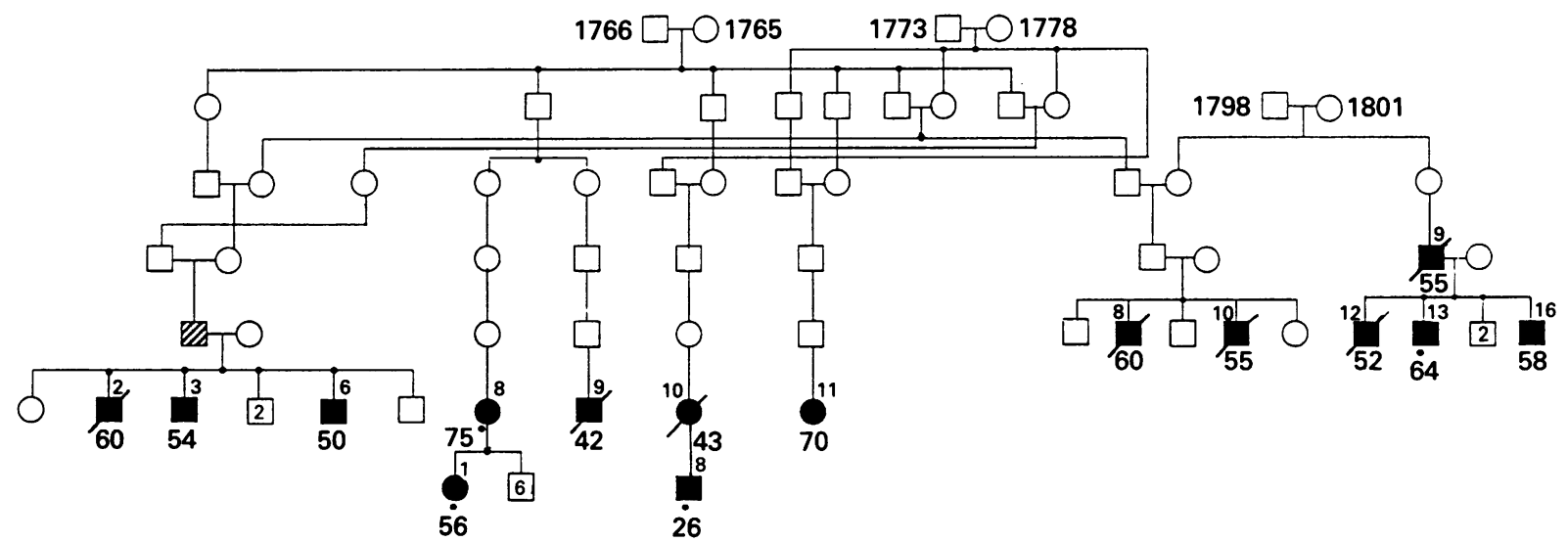

II

III

IV

VI

VII

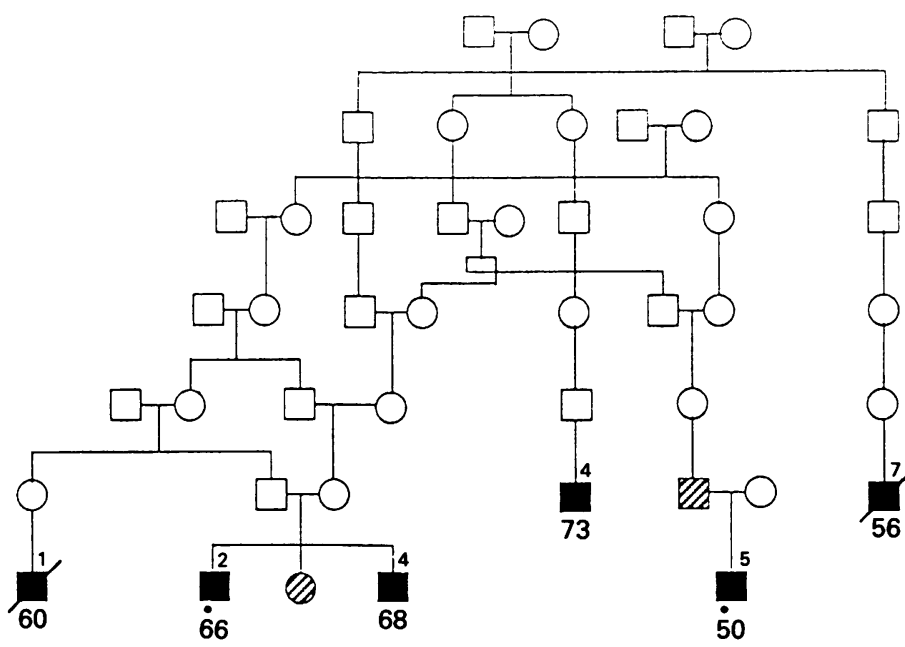

Figure 5 Pedigree PT-5. families can be noted. Two of them are linked to three nuclear families of today while the third is related to two of them. It is impossible to make any evaluation of the empirically most promising traces from the individual data presented here.

GENETIC ANALYSIS

The possible role of the sex of the transmitting parent

In 146 patients, it was possible to establish whether the gene was inherited from the paternal or the maternal side: 81 had inherited FAP from their father and 65 from their mother. Their mean ages of onset were 55.4 (SD 11.5) and 50.7 (SD 14.9) years, a difference which is statistically significant $\left(t_{145}=2.14, p=0.03\right.$ for the unpaired $t$ test). Also, out of 26 patients with onset below the age of 40, 20 had inherited the gene from their mother.

five in one family and three affected brothers out of five in another family. The father of the brothers in the second family had FAP as well. Considering ages of onset, all patients are close to the mean for Swedish FAP patients (table).

PT-4 involves five rather loosely related nuclear families. Three of them are alternatively connected to two separate key families born in the late 18th century. While ages of onset among three affected brothers in one of the nuclear families are close to the mean of the Swedish FAP population, the variability is striking in the rest of the patients in the pedigree. Not least marked are the differences between affected parents and children in this respect.

\section{$P T-5$}

Among the six patients in the pedigree, two are brothers and a third is their cousin. All three as well as two of the other patients involved in the pedigree have late ages of onset. The ancestral structure is complicated. Three separate key

Distribution of age of onset in some pedigrees.

\begin{tabular}{lcccc}
\hline Pedigree & No of patients & Range & Mean (SD) & Median \\
\hline SK-1 & 63 & $26-76$ & $49 \cdot 1(13 \cdot 8)$ & $47 \cdot 5$ \\
SK-2 & 10 & $46-75$ & $61 \cdot 1(9 \cdot 7)$ & $62 \cdot 5$ \\
SK-3 & 10 & $34-75$ & $58 \cdot 6(14 \cdot 8)$ & 62 \\
PT-2/PT-4 & 15 & $26-75$ & $54 \cdot 7(11 \cdot 8)$ & $55 \cdot 5$ \\
PT-5 & 6 & $50-73$ & $62 \cdot 2(8 \cdot 4)$ & 63 \\
\hline
\end{tabular}

\section{Anticipation in age of onset}

When describing the pedigrees, it was often observed that age of onset seemed to be lower in a later generation than in the previous one, particularly when the affected parent was the mother. We have studied 44 parent-offspring pairs with known ages of onset, 15 patients descended from an affected father and 29 from an affected mother. We defined anticipation as the parent's age of onset minus that of the offspring (in years).

While for offspring of affected fathers anticipation varied between -19 to 27 , meaning that at least some offspring had a later age of onset than their father, the range of anticipation for descendants of affected mothers was -1 to 32 , meaning that offspring of affected mothers mostly anticipate. Mean anticipation was 3.2 (SD 14.4) for offspring of affected fathers and 15.4 (SD 7.8) for offspring of affected mothers, a difference which was statistically significant $\left(t_{42}=3.68, p<0.001\right)$.

The correlation between sibs

The intraclass correlation coefficient, that is, the percentage of variability in age of onset which can be explained by the fact that patients are grouped in sibships, was calculated for 39 sibships (with more than one 
affected member). There was a mean number of 2.4 patients per sibship and the intraclass correlation coefficient was found to be 0.663 , showing a high degree of similarity among sibs.

The correlation between parents and offspring

The Pearson correlation coefficient, estimated from the 44 pairs of age of onset for each offspring and its affected parent, was 0.22 , which was not statistically different from zero $\left(t_{42}=1.47, p>0.05\right)$. This means that age of onset in offspring is not related (at least linearly) to age of onset in the affected parent.

This result seems in accordance with the anticipation patterns already described: large anticipation occurs when correlation of age of onset between parents and their progeny is close to zero, ${ }^{89}$ meaning that much variation is introduced by the allele of the unaffected parent.

\section{Discussion}

FAP in Sweden is concentrated to limited areas surrounding the two northern Swedish cities of Skellefteå and Piteå. Together with the hereditary character of the disease, this suggests that patients will be grouped in one or more big extended pedigrees. Swedish parish registers make it possible to illustrate the heredity, at least to a certain degree, as these historical archives normally start from the beginning of the 18 th century. Some extended pedigrees from both the Skelleftea and the Piteå areas have been presented. In spite of being geographically close, no genealogical links have been established between the two areas, at least back to the beginning of the 18th century, nearly 300 years ago. When observing these pedigrees, one general conclusion is that any mutation (either single or multiple) must have had its origin far back in time, probably the late mediaeval times.

Pedigrees are mostly used as mere illustrations of heredity and as diagnostic tools in clinical practice. However, pedigrees also illustrate more general patterns of heredity, for instance, concerning ages of onset among sibs and sibs in relation to their parents.

There seems to be a tendency for earlier ages of onset among patients descending from a carrier mother than from a carrier father. These results are even more striking than the ones found in the Portuguese FAP population. ${ }^{10}$ They could also be linked to anticipation patterns. Descendants of affected mothers seem to be more prone to anticipate age of onset than descendants of affected fathers. Anticipation has often been thought of as a result of ascertainment bias, ${ }^{8}$ since late onset offspring have not yet had the opportunity to manifest. It is possible that our sample of parent-offspring pairs, besides its small size, might also suffer from bias. However, the possible biases could not explain the sharp differences between the descendants of affected mothers and fathers. Moreover, similar results were found in a much bigger sample in the Portuguese FAP population. ${ }^{112}$

Pedigrees can also serve as tools or as empirical grounds for strategic selection of certain key groups of patients for further, deeper, and more far reaching analyses, with two aims in particular: one is to understand better why some patients have such a late onset, or some carriers may remain asymptomatic for their whole life; the other is to search for explanations for different expressions in age of onset when the disease is inherited from the mother or from the father.

This research was made possible by Fundaçao Gomes Teixeira da Universidade do Porto (A Sousa) and supported by the Familial Amyloidosis Patient Association (FAMY), Skellefteå, Sweden. Mrs Margit Lundmark is acknowledged for her skilful technical work.

1 Andrade C. A peculiar form of peripheral neuropathy. Brain 1952;75:408-27.

2 Araki S, Kurihara T, Tawara S, Kuribayashi T. Familial amyloidotic polyneuropathy in Japanese. In: Glenner GG, Costa PP, Freitas AF, eds. Amyloid and amyloidosis. GG, Costa PP, Freitas AF, eds. Amyloid and

3 Kito S, Itoga E, Kamiya K, Kishida T, Yamamura Y. Studies on familial amyloid polyneuropathy in Ogawa village, Japan. Eur Neurol 1980;19:141-51.

4 Saraiva MJM, Costa PP, Birken S, Goodman DS. Presence of an abnormal transthyretin (prealbumin) in Portuguese patients with familial amyloidotic polyneuropathy. Trans Assoc Am Physicians 1983;96:261-70.

5 Tawara $S$, Nakazato $K$, Kangawa $H$, Matsuo $T$, Araki S. Identification of amyloid prealbumin variant in familial amyloidotic polyneuropathy (Japanese type). Biochem Biophys Res Commun 1983;116:880-8.

6 Nakazato M, Steen L, Holmgren G, et al. Structurally abnormal transthyretin causing familial amyloidotic polyneuropathy in Sweden. Clin Chim Acta 1987;167:341-2.

7 Andersson R. Familial amyloidosis with polyneuropathy. A clinical study based on patients living in northern Swe-
cling clinical study based on patients living in north
den. Acta Med Scand 1976;suppl 590:1-64.

8 Penrose LS. The problem of anticipation in pedigrees of dystophia myotonica. Ann Eugen 1948;14:125-32.

9 Brackenridge CJ. Familial correlations for age at onset and age at death in Huntington's disease. $\mathcal{J}$ Med Genet 1972;9:23-32.

10 Sousa A, Coelho T, Sequeiros J. Parental transmission and age-of-onset in familial amyloidotic polyneuropathy (Portuguese type). In: Natvig J, Forre O, Husby G, et al, eds. Amyloid and amyloidosis. Dordrecht: Kluwer Academic Publishers, 1991:691-3.

11 Sousa A, Coelho T, Lobato L, Sequeiros J. Anticipation of age-of-onset in familial amyloidotic polyneuropathy (Portuguese type). In: Natvig J, Forre O, Husby G, et al, eds. Amyloid and amyloidosis.

12 Sousa A, Coelho T, Lobato L, Sequeiros J. Parental transmission and anticipation of age-of-onset in FAP-I. Am $\mathcal{F}$ Hum Genet 1991;49(suppl):A2757. 